\title{
La transigibilidad: un criterio incorrecto de arbitrabilidad
}

\author{
Leonardo Coronel Larrea* \\ Isabel Núñez Patiño**
}

Recibido/Received: 30/05/2019

Aceptado/Accepted: 31/07/2019

Sumario: 1. Introducción. 2. A manera de aclaración. 2.1 La existencia del convenio arbitral. 2.2 La validez del convenio arbitral. 2.2.1 Capacidad. 2.2.2 Manifestación de la voluntad libre de vicios. 2.2.3 Objeto y causa lícita. 2.3 La suficiencia del convenio arbitral. 3. Aproximación al problema: ¿qué es la arbitrabilidad? 3.1 Arbitrabilidad objetiva. 4. Algunos antecedentes. 5. La transigibilidad como regla de arbitrabilidad objetiva en el Ecuador. 6. El Derecho comparado y la arbitrabilidad objetiva. 6.1 Sobre el derecho francés. 6.2 Sobre el ordenamiento estadounidense. 6.3 El criterio de arbitrabilidad en Europa. 6.4 Criterio de Arbitrabilidad en Perú. 7. La transigibilidad y su problemática. 8. A manera de conclusión.

Resumen: El presente trabajo busca esclarecer ciertas confusiones que existen dentro del mundo del arbitraje. Asimismo, se plantea estudiar los problemas esenciales que se encuentran dentro del ordenamiento jurídico ecuatoriano, específicamente en cuanto al criterio de arbitrabilidad objetiva que el Ecuador establece: la transigibilidad. Dentro de este desarrollo se demostrará la arbitrabilidad, en sus dos facetas,

\footnotetext{
* Estudiante de pregrado del Colegio de Jurisprudencia de la Universidad San Francisco de Quito. Correo electrónico: leonardocoronellarrea@gmail.com / lcoronel@estud.usfq.edu.ec

** Estudiante de pregrado del Colegio de Jurisprudencia de la Universidad San Francisco de Quito. Correo electrónico: isabelitanp97@gmail.com / nnunezp@estud.usfq.edu.ec

L. Coronel Larrea \& I. NúÑez Patĩ̃o, "La transigibilidad. Un criterio erróneo de arbitrabilidad". Revista Ecuatoriana de Arbitraje, No. 10, 2019, pp. 155-186.
} 
subjetiva y objetiva. Con posterioridad, se centrará en la competencia ratione materiae y como esta se ha desarrollado en otras legislaciones. Finalmente, se expondrán los problemas que se encuentran enraizados en el criterio de transigibilidad, proponiendo que se deben realizar ciertos cambios siguiendo la perspectiva del arbitraje moderno.

Palabras Clave: arbitrabilidad, arbitrabilidad objetiva, transigibilidad, renunciabilidad, arbitraje, convenio arbitral, competencia, tribunal arbitral, ratione materiae, LAM.

\section{Transigibility: an incorrect arbitrability standard}

AвSTRAct: The present work seeks to clarify certain confusions that exist within the world of arbitration. Likewise, it is proposed to study the essential problems that are found within the Ecuadorian legal system, specifically with regard to the criterion of objective arbitration that Ecuador establishes: the transigibility. Within this development, arbitrability will be demonstrated, in both its facets, subjective and objective. Subsequently, it will focus on the ratione materiae competition and how it has developed in other legislations. Finally, the problems that are rooted in the transferability criterion will be exposed, proposing that certain changes must be made following the perspective of modern arbitration.

KeYWORDs: arbitrability, objective arbitrability, transigibility, renunciability, arbitration, arbitration agreement, competence, arbitral court, ratione materiae, LAM.

\section{INTRODUCCIÓN}

El nacimiento de Atenea, la diosa de la sabiduría, las ciencias, la justicia, la guerra, la civilización y la destreza, fue 
un hecho propio de la magnificencia de la favorita de Zeus. En un principio, el temor que sintió el regente del Olimpo por llegar a ser sobrepasado por uno de sus hijos fue tan grande que, cuando la madre de Atenea estaba en cinta, decidió tragársela para así evitar que su miedo se materializara1. Con el paso del tiempo, un malestar acongojó a Zeus, la molestia creció a tal punto que obligó a Hefesto que con un hacha abriese su cabeza, de donde nació Atenea "perfectamente armada y profiriendo su grito de guerra"².

Así como sucedió con Atenea, podemos ver que la justicia ordinaria presenta el mismo temor que Zeus: ser sobrepasada; en virtud de ello, trata de tragar a quien le profiere este miedo, el arbitraje. Es por esta razón, que se limita al sistema arbitral permitiendo que sólo determinados puntos puedan ser discutidos por este medio. Es indudable que el temor de ser superada involucra la creación de criterios inadecuados e inaplicables para limitar al sistema.

Sin embargo, así como Atenea nació armada y profiriendo el grito de guerra, el arbitraje ha nacido con principios que funcionan como armadura, protegiendo así su existencia, y la arbitrabilidad siendo su genialidad. Es claro que, con el paso del tiempo, esta genialidad irá incrementando para al final convertirse, como lo fue Atenea, en la favorita de su padre. El presente trabajo pretende realizar un análisis para (i) clarificar los momentos jurídicos del arbitraje. De esta manera, (ii) definir la arbitrabilidad y su división, para ahondar en la parte objetiva, materia del presente trabajo. Seguido a ello observaremos (iii) el criterio de arbitrabilidad en la legislación ecuatoriana; (iv) y en el derecho comparado. Finalmente, (v) se expondrá la necesidad de otro criterio de arbitrabilidad y la propuesta a seguir.

1. S. B. Pomeroy, Diosas, rameras, esposas y esclavas, Vol. 104, Ediciones AKAL, 2004.

2. Ibídem. 


\section{A MANERA DE ACLARACIÓN}

Es preciso aclarar determinados temas que han causado inconvenientes en el estudio de la Teoría General del Arbitraje. En primer lugar, es preciso tomar en cuenta el derecho por el que se rige el arbitraje, ya que de este se puede analizar, tanto la parte procesal y procedimental, así como la sustancial de la controversia ${ }^{3}$. Esto es de vital importancia, porque cada elemento del proceso debe ser corroborado tomando en cuenta los elementos legales para su correcta formación.

Tomando en cuenta este criterio, la primera norma que rige al proceso arbitral es la ley procesal o lex arbitrii. Esta es aquella que se enfoca en:

la definición y forma del acuerdo arbitral; la determinación de si las controversias pueden o no someterse a arbitraje; la constitución del tribunal arbitral y las causales de recusación de dicho tribunal, el derecho del tribunal arbitral a decidir sobre su propia jurisdicción; el trato equitativo entre las partes; la libertad de establecer normas procesales detalladas; las medidas cautelares; los escritos de demandas y contestación; las audiencias; [...] y, la forma y validez del laudo arbitral y el carácter definitivo del laudo, incluido el derecho a impugnarlo ante tribunales de justicia del lugar del arbitraje ${ }^{4}$.

En el caso ecuatoriano, sin lugar a dudas, se puede afirmar que la Ley de Mediación y Arbitraje (en adelante, LAM) cumple con todos los enfoques que se esperan de la lex arbitrii.

Como segunda norma, se encuentra la ley sustancial o de fondo de la controversia. Es claro que la tarea primordial del tribunal arbitral es solventar el conflicto con base en normas

3. K. H. BöCKStiegel, "Pespectives of future development in International Arbitrarion", en L. W. Newman \& R. D. Hill (Eds.), The Leading Arbitrators' Guide to International Arbitration, Juris Publishing Inc, 2004.

4. A. RedFern et al., Teoría y Práctica del Arbitraje Comercial Internacional, 4ta Ed., La Ley, 2007. 
generales y la subsunción de una base fáctica; para ello en cualquier relación jurídica debe existir un derecho de fondo, derecho aplicable o ley aplicable que regule esta ${ }^{5}$. Es decir, un ordenamiento jurídico que regule de manera general ciertos elementos de existencia, validez y eficacia de las instituciones legalmente reconocidas. En el Ecuador, la norma por antonomasia que contempla estos requisitos es el Código Civil.

Finalmente, las normas procedimentales, son aquellos reglamentos administrativos que se encargan del correcto funcionamiento del arbitraje, tanto en logística como en plazos y diligencias. Regularmente son aquellas normas utilizadas por los centros de arbitraje, cuando se habla de arbitrajes institucionales o administrados. Estas normas buscan que las partes procesales solo se enfoquen en litigar y no en los engorrosos pasos detrás del correcto funcionamiento de un proceso. Sin embargo, esto no quiere decir que un arbitraje ad-hoc no se pueda utilizar este tipo de normas, pues lo más común es que las partes acuerden seguir el lineamiento por una normativa procedimental ya establecida.

Ahora bien, en el arbitraje se debe tener en cuenta una división de momentos jurídicos, más que momentos temporales en sí mismos. Estos momentos son: (i) Existencia; (ii) Validez; y, (iii) Suficiencia. Cada uno de estos momentos tiene fundamental importancia pues repercuten y desencadenan los efectos esperados del convenio arbitral. Para terminar en el análisis de la competencia que tiene el tribunal arbitral. En este mismo sentido, las leyes del arbitraje convergen con este iter jurídico, toda vez que son las que establecen el cumplimiento o no de los requisitos necesarios para el correcto funcionamiento del arbitraje. A continuación, se procederá a revisar de manera breve a cada uno de estos para tener un panorama claro.

5. Compagnie d'Armement Maritime S.A. c. Compagnie Tunisienne de Navigation S.A., House of Lords, 1971. 


\subsection{La existencia del convenio arbitral}

La existencia engloba el análisis del nacimiento del convenio arbitral. Es decir, determinar si, efectivamente nació o no, a la vida jurídica un convenio por el cual las partes escogen el arbitraje como un método alternativo de solución de conflictos (en adelante, MASC). Convenio que genera "derechos potestativos a excepcionarse en la justicia ordinaria" en cada una de las partes ${ }^{6}$.

Para que se considere que existe el convenio se debe recurrir a la teoría general del negocio jurídico. En este sentido, cabe revisar tanto la lex arbitrii, como la ley sustancial del arbitraje. Los requisitos que la doctrina ha establecido son: (i) la voluntad; (ii) el objeto; y, (iii) las solemnidades que la ley exija ${ }^{7}$. Una vez que concurren todos estos elementos se ha de proceder a considerar como existente al convenio, y por ende se puede continuar hacia el análisis de validez.

Tomando en consideración cualquier ley procesal, es claro que el convenio arbitral debe ser por escrito ${ }^{8}$. El mismo criterio lo acogen la Ley Modelo de la Comisión de las Naciones Unidas para el Derecho Mercantil Internacional (en adelante, Ley Modelo CNUDMI) y la Convención de Nueva York (en adelante, CNY). El artículo II de la CNY establece que: "La expresión "acuerdo por escrito" denotará una cláusula compromisoria incluida en un contrato o un compromiso, firmados por las partes $[\ldots]^{\prime \prime 10}$. Esto apunta a que es un

6. Sobre el análisis de la naturaleza jurídica de los efectos del convenio arbitral, véase O. SANTOS DÁvalos, "Naturaleza jurídica de los efectos del convenio arbitral", Revista Ecuatoriana de Arbitraje, No. 8, 2016, pp. 117-142.

7. G. Ospina Fernández \& E. Ospina Acosta, Teoría General del Contrato y del Negocio Jurídico, $7 \mathrm{ma}$ Ed., Editorial Themis, 2018.

8. G. BoRN, International Commercial Arbitration: International and USA Spects, Transnational Publishers Inc., 2011.

9. Ley Modelo de la Comisión de las Naciones Unidas para el Derecho Mercantil Internacional sobre Arbitraje Comercial Internacional (2006), Art. 7.2.

10. Convención sobre el Reconocimiento y la Ejecución de las Sentencias Arbitrales Extranjeras (1958). Art. II. 
mandamiento de existencia de este negocio jurídico. Es decir, podría considerarse que es un requisito ad solemnitatem, toda vez que la voluntad de arbitrar debe ser reducida a escrito ${ }^{11}$.

Sin embargo, en la jurisprudencia y la doctrina actual, el requisito de contener la cláusula arbitral por escrito ha dado un vuelco totalmente interesante, tal es así que se han realizado varias interpretaciones acerca de la naturaleza jurídica de este requerimiento ${ }^{12}$. Es claro que:

[e]xiste una tendencia generalizada para aplicar el requisito 'por escrito' bajo la Convención [de Nueva York] de manera liberal, de acuerdo con el enfoque pro-ejecución y las actuales prácticas internacionales en donde los contratos se celebran por distintos medios. Una ampliación inflexible del requerimiento escrito [...] sería contradictoria a los usos comerciales corrientes y generalizados ${ }^{13}$.

De la misma manera, para CANTUARIAS y CAIVANO, la escrituración del convenio arbitral ha pasado de ser un requisito ad solemnitaten a ser un elemento ad probationem ${ }^{14}$. Es decir, una fórmula que ayude a corroborar los ámbitos de aplicación del tribunal arbitral una vez surgida la controversia, más no un ritual de formalidades que coarten la inevitabilidad del arbitraje.

Siguiendo esta línea, se podría afirmar que la existencia del convenio debe probarse, por lo que es necesario que conste algún tipo de fórmula en la que se haya materializado la concurrencia de las voluntades de las partes. La prueba ha de darse por escrito, como lo señala el artículo 5 de la $\mathrm{LAM}^{15}$. Esto conlleva a que siempre el convenio tenga como elemento existencial su escritura.

11. R. Von Ihering, Espíritu del derecho romano, Tomo III, 2da Edición, 1880.

12. H. GARCía LARRIVA, "Partes no signatarias del convenio arbitral: entre la realidad económica y la ficción jurídica", Revista Ecuatoriana de Arbitraje, No. 3, 2011, pp. 81-82.

13. ICCA, Guía ICCA para la interpretación de la Convención de Nueva York, 2013.

14. F. Cantuarias Salaverry \& R. J. Caivano, "La nueva ley de arbitraje peruana: un nuevo salto a la modernidad", Revista Peruana de Arbitraje, No. 7, 2008.

15. Ley de Arbitraje y Mediación, Art. 5, RO No. 147, 14/12/2006. 
Por lo tanto, la escritura del convenio puede darse de manera formal o informal, es decir, que no debe seguir un procedimiento específico para que sea considerado un convenio arbitral, lo que significa que basta su existencia tangible en cualquier medio sea físico o electrónico.

\subsection{La validez del convenio arbitral}

Dentro de la segunda fase o momento jurídico de este íter de análisis, se debe tener en cuenta un listado de requisitos necesarios para proceder a revisar su cumplimiento. El análisis se reduce a verificar si el convenio cumple con los requisitos de validez comunes a todos los negocios jurídicos que recoge la ley sustantiva del proceso arbitral [supra § 2.]. Como señala el artículo 1461 del Código Civil ${ }^{16}$, los requisitos son los siguientes: (i) la capacidad de las partes; (ii) la voluntad libre de vicios; (iii) objeto lícito; y (iv) causa lícita. A continuación, procederemos a revisar brevemente cada uno de ellos.

\subsubsection{Capacidad}

Como regla general se tiene que toda persona es capaz, así lo señala el artículo 1462 del Código Civil ${ }^{17}$, siendo necesario que se vean quiénes son las personas incapaces que declara la ley de manera taxativa. Este listado se compone por una división entre incapaces generales e incapaces especiales, comenzando con los incapaces generales se tiene una subdivisión que se compone por incapaces absolutos e incapaces relativos.

Por una parte, los incapaces absolutos son "los dementes, los impúberes y la persona sorda que no pueda darse a entender de manera verbal, por escrito o por lengua de señas"18,

16. Código Civil, Art. 1461, RO No. 46, 24/06/2005.

17. Ídem, Art. 1462.

18. Ídem, Art. 1463. 
teniendo como efecto que sus actos no surtan efecto alguno. Por otra parte, los incapaces relativos, que son "los menores adultos, los que se hallan en interdicción de administrar sus bienes y las personas jurídicas" ${ }^{19}$, tienen la posibilidad de que sus actos surtan efecto alguno bajo ciertas circunstancias y cuando la ley lo permita. En el caso de los incapaces especiales nos encontramos con la "prohibición que la ley ha impuesto a ciertas personas para ejecutar ciertos actos" ${ }^{20}$.

\subsubsection{Manifestación de la voluntad libre de vicios}

Cuando se habla de que la voluntad se encuentre libre de vicios se trata de que el consentimiento puede verse afectado por el error, la fuerza y el dolo como lo señala el artículo 1467 del Código Civil ${ }^{21}$. El error vicia el consentimiento cuando recae sobre la identidad de la cosa, sobre una cualidad de la cosa, sobre el negocio jurídico que se está celebrando, sobre la identidad de la persona con la que se contrata cuando haya sido la causa principal por la que se contrató.

Cuando se trata de la fuerza, existe vicio cuando se ha producido una "impresión fuerte en una persona de sano juicio, tomando en cuenta su edad, sexo y condición" ${ }^{22}$, y posee el temor de verse expuestos a ésta la persona misma, "su cónyuge o alguno de sus ascendientes o descendientes, a un mal irreparable y grave" ${ }^{23}$. El dolo se ve restringido a la acción de una de las partes y que sea evidentemente manifiesto que no ser por él no se hubiese contratado, caso contrario el dolo no produce vicio en el consentimiento, como lo señala el artículo 1474 del Código Civil ${ }^{24}$.

\footnotetext{
19. Ibídem.

20. Ibídem.

21. Ídem, Art. 1467.

22. Ídem, Art. 1472.

23. Ibídem.

24. Ídem, Art. 1474.
} 


\subsubsection{Objeto y causa lícita}

En el caso del objeto es necesario que este se encuentre dentro del comercio jurídico, que se determine o sea determinable, en el caso de que sea una cosa, pero so se trate de un hecho se debe ver su posibilidad física y moral, es decir, que sea realizable y que no vaya contra el orden público y las buenas costumbres. En cuanto a la causa, se debe entender "el motivo que induce al acto o contrato" ${ }^{25}$ la cual no es contraria a las buenas costumbres o al orden público; su importancia radica en que no puede existir obligación sin ella, pero no es necesario que se la exprese.

\subsection{La suficiencia del convenio arbitral}

En esta tercera fase se analiza la suficiencia del convenio para que surta los efectos deseados por las partes. Por ello es necesario sacar a relucir el principio de kompetenz-kompetenz. Este se entiende como "la potestad que tiene el tribunal arbitral para decidir sobre su propia competencia, al igual que, sobre las excepciones referentes a la existencia o a la validez del acuerdo de arbitraje" ${ }^{\prime 26}$. Su origen se da en una sentencia emitida por el Tribunal Superior de la República Federal de Alemania en el año 1955. En esta se estipulaba que se debía conceder a los árbitros la potestad tanto de establecer el "alcance del acuerdo arbitral que se somete a su conocimiento" ${ }^{27}$, como conocer su propia competencia y autoridad.

Así mismo, este principio se dio a relucir en la sentencia emitida, en el caso First Options of Chicago Inc. c. Kaplan, del año de 1995 por la Corte Suprema de los Estados Unidos. En este caso sucedió que, tras la caída de la bolsa de 1985, First Options

25. Ídem, Art. 1483.

26. S. Vargas, C. Figuera \& J. F. Puertas Barahona, "El Principio kompetenz-kompetenz del arbitraje comercial internacional en la jurisprudencia del Tribunal Supremo de Justicia Venezolano". Revista de la Facultad de Jurisprudencia RFJ, 2018.

27. J. Fernández, R. Arenas \& P. A. De Miguel, Derecho de los negocios internacionales, Iustel, 2007. 
demandó a Manuel Kaplan, a su esposa y a su compañía MK Investments Inc. él pagó inmediatamente la deuda que tenían. Al momento en que First Options demandó por el pago insatisfecho, se procedió a solicitar el arbitraje en un panel de la Bolsa de Valores de Filadelfia, basándose en acuerdos de entrenamiento, que regían el funcionamiento de las deudas de Kaplan, su esposa y MKI. Sucedió que MKI había firmado un documento en el que se encontraba un acuerdo de arbitraje, cosa que no habían hecho los Kaplans, por lo que presentaron objeciones.

Estas objeciones tenían que ver con que el desacuerdo que mantenían los Kaplan con First Options no era arbitrable, por lo que se procedió a decidir sobre los méritos de la disputa, resultando en que los árbitros decidieron a favor de First Options. En última instancia, el Tribunal de Apelaciones revocó el laudo, al considerar que la disputa no era arbitrable. La pregunta que se encontró en este caso fue, si la arbitrabilidad de la controversia se encuentra sujeta a revisión independiente por parte de los tribunales y si deben los tribunales de apelación aplicar un estándar de abuso de discreción al revisar los tribunales de arbitraje que sostienen los laudos.

La respuesta que dio la Corte para esto fue que la capacidad de arbitraje del presente caso estaba sujeta a una revisión independiente por parte de los tribunales y que, en lugar de un estándar especial de abuso de discreción, los tribunales de apelación deberían aplicar una normativa común al revisar las decisiones que provienen de los tribunales de distrito que sostienen los laudos arbitrales, concluyendo específicamente que "debido a que los Kaplans no acordaron claramente someter la cuestión de la arbitrabilidad al arbitraje, el Tribunal de Apelaciones acertó al encontrar que la arbitrabilidad de la disputa de Kaplan-First Options estaba sujeta a una revisión independiente por parte de los tribunales" ${ }^{28}$. A pesar de haber

28. First Options of Chicago, Inc. c. Kaplan, Corte Suprema de Estados Unidos, 1995. 
anulado el laudo en este caso, la Corte Suprema de Justicia mantiene y recalca los efectos del kompetenz-kompetenz, toda vez que el tribunal arbitral el primer encargado para dirimir su propia competencia.

Según lo establece Giuseppe CHIOvendA, "la competencia es el conjunto de las causas en que puede ejercer, según la ley, o jurisdicción, y en otro, se entiende por competencia esta facultad del tribunal considerada en los límites en que le es atribuida" ${ }^{\prime 29}$. Así mismo la Corte Suprema de Chile establece que:

Las reglas de competencia se orientan a determinar cuál será el tribunal competente para conocer de un asunto determinado, pudiendo reconocerse aquellas de carácter general, aplicables a toda clase de materia y tipo de tribunales -de radicación; del grado o jerarquía; de extensión; de prevención o inexcusabilidad y de ejecución y las especiales, que dicen relación con la competencia de los tribunales que integran el Poder Judicial, pudiendo a su vez distinguirse entre estas, las relativas a la competencia absoluta, esto es, la cuantía, la materia y el fuero personal, y las de competencia relativa, que son aquellas que tienen por objeto determinar de entre tribunales de una misma jerarquía o categoría, cuál de ellos será el competente para conocer de un asunto determinado ${ }^{30}$.

La competencia no sólo se debe divisar dentro de la justicia ordinaria, sino que también es necesario conocer cómo se comporta en los medios alternativos de resolución de conflictos, específicamente en el arbitraje. En este punto, converge el análisis de la arbitrabilidad, como factor común a todo proceso arbitral.

\section{Aproximación Al PROBlema: ¿QUÉ es la ARbitrabilidad?}

La arbitrabilidad es aquel criterio legal que busca el interés general de la resolución de controversias, y está íntimamente

29. G. Chiovenda, Instituciones de Derecho Procesal Civil, Vol. II, Revisto, p. 175.

30. Adm. de Inv. y Sup. Unimarc S.A. con Empresa de Servicios Sanitarios del Bío Bío S.A., Interagro Comercio y Ganado S.A, Corte Suprema de Chile, Sentencia, 9/12/2013. 
relacionado con los asuntos (materia) que pueden ser solventados y partes (persona) que pueden ser juzgadas por arbitraje ${ }^{31}$. Es decir, es aquella "susceptibilidad del objeto de las pretensiones de las partes de ser resuelto en un arbitraje; [y] los criterios de la arbitrabilidad de las partes de ser resuelto en un arbitraje" ${ }^{\prime 32}$. En este sentido existen dos tipos de arbitrabilidad, la doctrina realiza la siguiente división: (i) arbitrabilidad subjetiva; y, (ii) arbitrabilidad objetiva ${ }^{33}$.

La arbitrabilidad subjetiva se da en razón de las personas, mientras que la objetiva se da de acuerdo a las materias comprendidas. Según la Ley Modelo CNUDMI, existe una sola arbitrabilidad, esto es, la objetiva. Centrándonos en esta división se explicará brevemente la arbitrabilidad subjetiva, pues no es materia del presente trabajo. El criterio de arbitrabilidad subjetiva se refiere a "controversias en las que ciertos individuos o entidades son considerados incapaces para remitir sus disputas al arbitraje por razón de su estatus o funciones" ${ }^{\prime 34}$. En síntesis, es lo que se conoce como competencia ratione personae, básicamente la facultad del tribunal arbitral para pronunciarse y poder juzgar a determinadas partes cobijadas por el convenio arbitral.

\subsection{Arbitrabilidad objetiva}

Al enfocarse en la arbitrabilidad objetiva, determinar o no las materias susceptibles de ir a arbitraje, es a todas luces, decisión de cada Estado. Es decir, este criterio depende del derecho doméstico de cada país, pues como se puede ver en el artículo 1(5) de la Ley Modelo CNUDMI se prescribe que:

31. E. Gaillard \& J. Savage, Fouchard Gaillard Goldman on International Commercial Arbitration, Kluwer Law International, 1999.

32. Collantes et al., Diccionario terminológico del arbitraje nacional e internacional (Comercial y de Inversiones), Lima, 2011.

33. E. León Robayo, "Perspectiva y arbitrabilidad de la Convención Interamericana de Arbitraje Comercial Internacional”, Opinión Jurídica, Vol. 11, No. 22, 2012.

34. Ibídem. 
“[e]sta ley no afectará a ninguna otra ley en virtud de la cual determinadas controversias no sean susceptibles de arbitraje o se pueda someter a arbitraje únicamente de conformidad con disposiciones que no sean las de la presente ley"35. En algunas legislaciones la arbitrabilidad de una controversia coincide con "la disponibilidad que tienen las partes respecto del objeto"36, mientras que, en otras, "resulta arbitrable cualquier interés patrimonial, sin que esta noción coincida con la naturaleza disponible de los derechos ${ }^{\prime \prime 37}$.

La arbitrabilidad objetiva trata de "determinar si el contenido mismo del asunto puede ser objeto de arbitraje y, por ende, conocido por árbitros" ${ }^{\prime \prime 3}$. En otras palabras, se ve las materias que por mandato del legislador han sido reservadas para ser revisadas solamente por los jueces nacionales.

En la actualidad no existe un consenso general sobre las materias que no son arbitrales, toda vez que varían de jurisdicción en jurisdicción ${ }^{39}$. Sin embargo, se han determinado algunas tendencias respecto a este punto. Por ello en necesario seguir a Cárdenas en este aspecto, ya que sostiene que: (i) el arbitraje que no se encuentra relacionado con litigios patrimoniales es improcedente; (ii) los litigios patrimoniales cuya decisión se reserva a las entidades públicas, considerando los intereses existentes y la plausible afectación que se pudiese ocasionar a terceros; (iii) las controversias dentro de procesos concursales referidas a las decisiones o actuaciones propias del mismo no son arbitrables; (iv) derechos que poseen una indisponibilidad absoluta; y, (v) el hecho de que se aplique una norma de orden público

35. Ley Modelo de la CNUDMI, N. 9, Art. 5.

36. E, Mereminskaya, "La relación entre la arbitrabilidad y el orden público en la jurisprudencia comparada", Iuris Dictio: Arbitrabilidad y orden público, 2007.

37. Ibídem.

38. Ibídem.

39. F. González De Cossío, Arbitraje, 2da Ed., Editorial Porrúa. 
no afecta la posibilidad de $\operatorname{arbitrar}^{40}$. Esta bifurcación se da debido a que en algunos países se puede arbitrar y en otros se encuentra prohibido hacerlo.

De hecho, revisando estas tendencias actuales se puede ver que tiene dos dimensiones, la primera es que puede ser vista como "una restricción al poder de comprometer o una incapacidad legal derivada de las circunstancias referentes a la naturaleza del litigio [y la segunda dimensión es como] un defecto del convenio arbitral, condición de validez del mismo" ${ }^{\prime 1}$. La primera de estas tiene que ver con el fondo del conflicto, por lo que es "la lex causae o ley aplicable al fondo del litigio la que debe determinar si una determinada categoría de litigios es arbitrable y en qué circunstancias" 42 , mientras que la segunda de ésta tiene que ver con el sometimiento de las "consideraciones a la ley rectora de la validez por razones de fondo del convenio arbitral" ${ }^{43}$.

A continuación, se revisará algunos antecedentes de la legislación ecuatoriana, además de verificar cómo es el criterio dentro del país y cómo se ha manejado la arbitrabilidad objetiva en legislaciones extranjeras; después se analizará el criterio propio en cuanto al tema.

\section{Algunos antecedentes}

El sistema arbitral ecuatoriano ha tenido un gran desarrollo en los últimos años. En un principio, se utilizaban de los lineamientos establecidos en la antigua Ley de Arbitraje Comercial, la derogada Ley de la Función Judicial ${ }^{44}$

\footnotetext{
40. M. VÁsquez Palma, "La arbitrabilidad objetiva: Aspectos históricos, modernas tendencias en derecho comparado y ubicación en el escenario legislativo chileno”, Revista Ius Et Praxis, 2016.

41. Ibídem.

42. Ibídem.

43. Ibídem.

44. Véase, Ley Orgánica de la Función Judicial, RO No. 636, 11/09/1974.
} 
(en adelante, LFJ) y el Código de Procedimiento Civil ${ }^{45}$. Este desarrollo termina con la expedición de la $\mathrm{LAM}^{46}$, que ha ido evolucionando con la práctica durante estos años.

El criterio de arbitrabilidad que antes se utilizaba estaba en el artículo 87 de la LFJ. Este prescribía que: “[p]ueden decidirse por árbitros solo las controversias sobre bienes o derechos que, siendo renunciables, puedan trasmitirse por acto entre vivos ${ }^{\prime 47}$. Es decir, las materias susceptibles de arbitraje eran aquellas que las partes podían renunciar a su arbitrio. Con la expedición de la LAM, el criterio fue modificado por el de transigibilidad de la materia. En palabras de ANDRADE CEVALLOS, "al haberse derogado esa norma la posibilidad desometer a arbitrajelas controversiasseha ampliado" ${ }^{\prime 4}$. Sin embargo, este criterio ha generado problemas en la actualidad, a pesar de encontrarse derogado. Toda vez que existen confusiones respecto a este tema, complicando una vez más el sistema.

Por esta razón, este artículo pretende ahondar en un análisis de arbitrabilidad que aclare, en principio, esta problemática. Para poder proponer un diferente lineamiento que guíe y sea directriz del arbitraje en Ecuador.

Es evidente que, tras veinte y dos años de la expedición de la LAM, han existido varios temas de análisis y de desarrollo que han surgido con la evolución práctica del sistema arbitral. Sin embargo, queda claro que varios temas todavía deben ser analizados y reformados para evitar futuros inconvenientes como los que han aparecido durante los últimos años. Ahora es el turno de analizar el criterio de arbitrabilidad, y determinar si el criterio acogido por el ordenamiento jurídico es el correcto o cual sería el cambio que se debe realizar.

45. Véase, Código de Procedimiento Civil, RO No. 58, 12 /07/2005.

46. Véase, Ley de Arbitraje y Mediación, N. 15.

47. Véase, Ley Orgánica de la Función Judicial, N. 44, Art. 87.

48. M. Andrade Cevallos, "Ley de Arbitraje y Mediación Transigible y Arbitraje en Equidad", Revista Ecuatoriana de Arbitraje, No. 4, 2012. 


\section{LA TRANSIGILIDAD COMO REGLA DE ARBITRABILIDAD OBJETIVA EN EL ECUADOR}

Se ha demostrado que el criterio de arbitrabilidad objetiva dependerá de la legislación de cada país ${ }^{49}$. Es decir, el legislador es el único que puede determinar la norma a seguir, al ser un tema de política pública, tanto legislativa como judicial ${ }^{50}$. En este sentido, el Ecuador ha optado como la regla de competencia ratione materiae que los árbitros deben seguir es la de aquellas materias susceptibles de transacción.

Así lo reconoce la Constitución (en adelante, CRE) en su artículo 190, el cual prescribe que: “[s]e reconoce el arbitraje, la mediación y otros procedimientos alternativos para la solución de conflictos. Estos procedimientos se aplicarán con sujeción a la ley, en materias en las que por su naturaleza se pueda transigir" 51 (énfasis añadido). En el mismo sentido, la LAM en su artículo 1 manda que: "[e]l sistema arbitral es un mecanismo alternativo de solución de conflictos al cual las partes pueden someter de mutuo acuerdo, las controversias susceptibles de transacción [...]" ${ }^{52}$ (énfasis añadido).

De esta manera, el criterio acogido por el ordenamiento jurídico ecuatoriano remite el análisis al contrato de transacción recogido en el CC. El artículo 2348 CC la define como: "[...] un contrato en que las partes terminan extrajudicialmente un litigio pendiente, o precaven un litigio eventual" ${ }^{53}$. Asimismo, el artículo 2349 determina que: "No puede transigir sino la persona capaz de disponer de los objetos comprendidos en la transacción" ${ }^{24}$. En este punto, el análisis se complica y puede ser mal interpretado, toda vez que entra en juego el tema de la libre disponibilidad.

49. R. J. Caivano, "Arbitrabilidad y Orden Público", Foro Jurídico, No. 12, 2013.

50. O. Marzorati, "Los límites del acuerdo arbitral", Revista La Ley, 2010.

51. Constitución de la República del Ecuador, Art. 190. RO No. 449, 20/10/2008.

52. Ley de Arbitraje y Mediación, N. 15, Art. 1.

53. Código Civil, N. 16, Art. 2348.

54. Código Civil, N. 16, Art. 2349. 
Por un lado, el término transigibilidad puede ser entendido como aquel que requiere concesiones dentro de las partes ${ }^{55}$, es decir debe existir la intención de negociar o disponer de un derecho que se tiene. En este punto, es preciso recordar el criterio previo de arbitrabilidad en el Ecuador [supra $\S 4$.]. Es decir, la renunciabilidad como lineamiento para determinar materia arbitrable. Debido a que, se han podido evidenciar confusiones entre los términos transigibilidad y renunciabilidad. A pesar de que es claro que transigibilidad no es sinónimo de renunciabilidad ${ }^{56}$. A criterio propio, se entiende que la confusión se da por el antiguo sistema y la falta de actualización con el avance normativo. Por lo tanto, es preciso aclarar y distinguir ambos términos para evitar confusiones incoherentes con el actual desarrollo arbitral. Bajo este presupuesto, es necesario definir y explicar cada termino sujeto a confusión.

Por otro lado, el termino renuncia puede ser definido como "la dejación o reducción voluntaria y consciente patrimonial que se hace de una cosa, un derecho, una acción o un privilegio que está reconocido a su favor ${ }^{57}$. De la misma manera, el Diccionario de la RAE define el término renuncia como: "[h]acer dejación voluntaria, dimisión o apartamiento de algo que se tiene, o se puede tener" ${ }^{25}$. Estas definiciones dejan como conclusión que renuncia es el hecho por el cual una persona se aparta o abandona un derecho existente dentro de su patrimonio.

Es preciso aclarar que se utilizan estas definiciones por una ausencia de definición legal. Pues en palabras de SANTOS DÁvalos:

Nuestro Código Civil no contiene definición alguna de renuncia. Empero, su artículo 11 dispone que los derechos

55. G. Cabanellas, Diccionario Enciclopédico de Derecho usual, Heliasta, 2008.

56. M. Andrade Cevallos. N. 48.

57. G. Cabanellas, N. 55.

58. Real Academia Española, Diccionario, <https://dle.rae.es/?id=Vyqz9zm>, (21/08/2019). 
son renunciables por regla general. Son irrenunciables solo aquellos derechos que la ley ha declarado como tales y aquellos que no miran al solo interés del renunciante ${ }^{59}$.

En conclusión, transigibilidad no es lo mismo que renunciabilidad. Por lo tanto, es inconcebible que existan confusiones respecto de estos términos. Actualmente, el criterio de arbitrabilidad objetiva es la transigibilidad de la materia. Sin embargo, de ello, esta postura instaurada en la CRE y la LAM genera problemas, por lo que se la criticará más adelante y se propondrá un cambio dentro de esta regulación.

\section{El DERECHO COMPARADO Y LA ARBITRABILIDAD OBJETIVA}

A nivel mundial la arbitrabilidad se puede ver desde diferentes ópticas. Por lo tanto, es preciso ahondar en los diferentes criterios de arbitrabilidad que pueden existir en el derecho comparado.

En este sentido, existen varias posturas respecto de cómo delimitar la arbitrabilidad objetiva. La primera es la propuesta por VAN DEN BERG, quien manifiesta que la materia debe ser delimitada bajo el derecho del foro o la lex fori; es decir que la ley sustancial delimitará qué es y qué no es arbitrable ${ }^{60}$. En contraste con esta postura se puede observar aquellos quienes sostienen que la lex arbitrii o ley procesal es la que determina la arbitrabilidad de los diferentes asuntos que ante ella se presenten ${ }^{61}$. Asimismo, está aquella posición que mezcla las dos anteriores, donde es obligación del árbitro o del juez realizar un análisis utilizando la lex fori y la lex arbitrii a la vez.

59. O. Santos Dávalos, N. 6.

Sobre el artículo 11 del Código Civil: "[p]odrán renunciarse los derechos conferidos por las leyes, con tal que sólo miren al interés individual del renunciante, y que no esté prohibida su renuncia".

60. A. J. Van Den Berg, The New York Convention Guide.

61. G. Asken, "American Arbitration Accession Arrives in the Age of Aquarius: United Estates Implements United Nations Convention on the Recognition and Enforcement of foreign Arbitral Awards", Southwestern University Law Review, 1971. 
De la misma manera, para un análisis correcto de la competencia ratione materiae, se debe sumar a cualquiera de los tres criterios antes mencionados, lo que JAN PAULSSON describe como la presunción en favor de arbitrabilidad, que tiene como objetivo beneficiar y tener la puerta abierta para que cualquier controversia pueda ser arbitrable ${ }^{62}$, siempre y cuando las partes no interpongan una excepción de inarbitrabilidad. Finalmente, se debe utilizar los criterios de orden público internacional ${ }^{63}$, para así evitar cualquier violación de esta institución del derecho, al ser el límite efectivo de la voluntad en el ámbito internacional.

Una vez establecidas las diferentes formas en las que se puede encontrar la arbitrabilidad en el derecho comparado, es preciso ahondar en varios sistemas para observar cómo se ha presentado la evolución de la competencia ratione materiae. Para ello, se analizará el ordenamiento francés, con posterioridad el estadounidense, un breve comentario del Derecho europeo y finalmente el enfoque de la región representado por la legislación peruana.

\subsection{Sobre el derecho francés}

En primer lugar, se analizará el criterio adoptado por Francia. En este caso, la legislación y jurisprudencia dan soluciones conforme el desarrollo práctico del derecho en el país. El Código Civil francés en su artículo 2059 establece que "[t]odas las personas pueden establecer compromiso sobre los derechos que se encuentran bajo su libre disposición" ${ }^{\prime}$. Es decir, se acoge el criterio de la disponibilidad de derechos reglamentado por las prohibiciones subsiguientes.

62. J. Paulsson, "Still Through a Glass Darkly", ICC International Court of Arbitration Bulletin: Arbitration in the Next Decade, Special Supplement, 1999.

63. E. Gaillard \& J. Savage, N. 31.

64. Código Civil Francés, Art. 2059. 
Toda vez que el artículo siguiente establece como excepciones que "no se pueden comprometer en árbitros las cuestiones de estado y de capacidad de personas, las relativas al divorcio y la separación de cuerpos o las que interesen a las colectividades públicas y los establecimientos públicos y, más generalmente, todas las materias que interesen al orden público" (énfasis añadido) ${ }^{65}$.

Esto lleva a ver que existe cierta resistencia a que la arbitrabilidad objetiva sea mucho más abierta en su legislación. Sin embargo, la jurisprudencia más reciente demuestra lo contrario. Debido a que se está optando por permitir que exista el arbitraje en asuntos de interés de orden público.

Tal es el caso 2004/19673 de la Corte de Apelaciones de Paris, del año $2006^{66}$. En este proceso se analizó un contrato que se había firmado en 1993 con el artículo 82 del Tratado CE. Así también, versó sobre la relevancia que tenía un contrato firmado en 1991 por las partes. El último contrato fue ampliamente discutido pero su validez nunca se cuestionó en el proceso arbitral. Dentro de las objeciones respecto a la arbitrabilidad de la materia se utilizaban mecanismos guiados a la incompetencia del tribunal arbitral por inmiscuirse en temas de orden público. Se decía que al tratarse de normas de orden público el tribunal arbitral no debía pronunciarse, ni emitir criterio alguno.

Los contratos trataban temas que tienen intereses de orden público, pues se tocaron materias como derecho penal, y derecho de competencia, que por regla general son de orden público. A pesar de aquello, no se vio impedimento en este caso para que se proceda a arbitrar. A la final la Corte rechaza la solicitud de instancia, por los artículos 81 y 82 del Tratado Ce; y confirma las decisiones del 15 de septiembre de 2004 que otorgan la ejecución al laudo del CCI 11018/DB/TE de 5 de

65. Ibíd, Art. 2060.

66. Caso CT0051, Corte de Apelaciones de París, Sentencia, 23/03/2006. 
noviembre de 2002 y 11018/TE/MW / AVH de 28 de julio de 2004. De esta manera se reafirma el cambio de la forma de pensar del ordenamiento jurídico francés, pues es evidente una ampliación respecto al control de la autonomía de la voluntad de las partes por el orden público.

Asimismo, en el caso 2016/22215 de la Corte de Casación, se presenta una disputa sobre una cláusula arbitral contenida en una sociedad civil. Esta manifestaba que se podía arbitrar cualquier disputa que pueda surgir en relación con el contrato suscrito por las partes, incluyendo los valores de reembolso, las acciones del socio que está retirado o excluido, o la valoración de las acciones. El problema con el que se dio respecto de la materia fue que se incumplió con lo que designaba el artículo 1843.4 del Código Civil, el cual expresamente establece que la cesión de los derechos de una sociedad y el valor de los derechos deberán determinarse de acuerdo a la forma en que se determine la ley y la cesión de derechos se dio conforme a lo que establecían los estatutos de la sociedad, por lo que se iba en contra de lo que establecía el legislador para la protección de derechos de terceros. Sosteniendo que el carácter de orden público no excluye la posibilidad de arbitraje de la disputa, por lo que se decide que estos puntos eran parte del examen de la jurisdicción arbitral.

Tanto la legislación como la jurisprudencia tienen una cohesión. Por un lado, es notorio que los árbitros pueden decidir el incumplimiento y la validez de contratos que tengan que ver en parte con cuestiones que tengan intereses de orden público y la legalidad de la conducta de las partes. Por otro lado, se ve que el legislador ha decidido qué temas se pueden considerar de orden público, pues estos temas serán tratados por la justicia ordinaria únicamente. 


\subsection{Sobre el ordenamiento estadounidense}

Como señala CAIVANO, se ha visto un adelanto en el concepto que manejan de arbitrabilidad ${ }^{67}$. En cuanto a su legislación interna, la más importante es la Ley Federal de Arbitraje (en adelante, FAA), la cual establece el régimen correspondiente para el arbitraje nacional e internacional. La FAA se encuentra compuesta por tres capítulos, los cuales fueron promulgados en diferentes fechas y tratan materias distintas. El primer capítulo de esta fue promulgado, en el año de 1925, y prevé tanto la validez como la aplicabilidad de los acuerdos de arbitraje internacional; el segundo, en el año de 1970, y versa sobre la aplicación de la Convención de Nueva York; y, el tercer capítulo en 1990 y trata sobre la Convención Interamericana.

Los dos últimos capítulos fueron enmiendas que se realizaron a la FAA original, con el objetivo de tener un sistema más eficaz y estable, para que Estados Unidos se vea como un ambiente apto para el comercio y la inversión ${ }^{68}$. En cuanto a las leyes estatales, cada estado posee una ley arbitral propia, aunque algunas puedan ser similares.

En cuanto a la jurisprudencia se tienen casos como el de Scherk c. Alverto- Culver Co., el cual trataba sobre derechos relativos a marcas sobre determinados productos que se veían cuestionados, violando así la ley sobre transacciones de crédito. La Corte Suprema consideró que una "cláusula arbitral incluida en un contrato internacional debe ser respetada en los términos de la FAA, si no existe alguna de las razones de las que pueda derivar la nulidad de cualquier otro contrato" ${ }^{\prime 69}$. La Corte rememoró que las cláusulas de selección del foro son condición necesaria para los contratos de carácter internacional, para poder evitar la "incertidumbre nacida de las múltiples

67. R. J. Caivano, N. 49.

68. Ibídem.

69. Ibídem. 
normas de conflicto que puedan ser aplicables y el peligro de que la controversia sea dirimida en un foro hostil a una de las partes o poco familiarizado con el tema a resolver"70. Así la Corte decidió que permitiría a una de las partes desconocer la promesa de resolver los conflictos por la vía arbitral. De lo visto se puede concluir que el rango de arbitrabilidad de los tribunales norteamericanos es bastante amplia y no se ve como obstáculo a las normas de orden público, considerando el motivo primigenio de la FAA, que se ha caracterizado por ser pro- arbitraje. El criterio con el que se maneja la arbitrabilidad es basado en los derechos patrimoniales, aquellos que se enfocan, sobre todo en el comercio y las inversiones, más los ámbitos como los que tratan puntos esenciales como la muerte, se mantienen en el ámbito de las materias que le competen únicamente a la justicia ordinaria.

De lo visto se puede concluir que el rango de arbitrabilidad de los tribunales norteamericanos es bastante amplia y no se ve como obstáculo a las normas de orden público, considerando el motivo primigenio de la FAA, que se ha caracterizado por ser proarbitraje.

\subsection{El criterio de arbitrabilidad en Europa}

En el caso de Europa se puede ver que existe una mayor cohesión en cuanto al rango de lo que es arbitrable, sobre todo en lo que "concierne a contratos comerciales internacionales"71, siendo pocas las áreas que no se pueden resolver por la vía arbitral. Por consiguiente, el sistema europeo propende la resolución de controversias por medio del arbitraje, siendo utilizado un criterio en extremo amplio para incentivar el avance y la evolución del proceso arbitral como MASC efectivo.

70. Ibídem.

71. Ibídem. 


\subsection{Criterio de arbitrabilidad en Perú}

En el caso de Perú encontramos en su legislación la existencia de una ley especializada, la cual es el Decreto Legislativo No. 1071, a su vez el Reglamento de Arbitraje del Centro de Conciliación y Arbitraje de la Superintendencia de Entidades Prestadoras de Salud; Estatuto y reglamento Arbitral del Centro de Arbitraje Popular del Ministerio de justicia, así como la Tabla de Aranceles, la Ley No. 29280, en su cuadragésima novena Disposición Final.

Dentro del Decreto Legislativo que norma el arbitraje en el artículo $2^{72}$ se ven las materias que son susceptibles de arbitraje, y se puede notar que arbitrabilidad es mucho más extensa que en otras legislaciones, pues el artículo no da una lista taxativa, sino que es más bien abierta e incluso reenvía a otras normas como es el caso de los acuerdos internacionales. Sumado a lo ya dicho se ve que desde el principio se tiene como base la inevitabilidad del arbitraje.

Legislativamente se ha optado por una forma monista en la que se trata de manera única al arbitraje, indistintamente si es nacional o internacional, lo que refleja que se encontraría una mayor eficiencia para los usuarios del arbitraje y una mayor seguridad jurídica, pues existe un conocimiento claro de cómo se ha de proceder. En cuanto a la colaboración y control judicial, se puede ver que se da preferencia a la asistencia del juez subespecializado en lo comercial, por sobre el civil, siendo este último la alternativa, como se puede ver en el artículo 8 del Decreto Legislativo No. 1071.

72. Artículo 2.- Materias susceptibles de arbitraje.

1. Pueden someterse a arbitraje las controversias sobre materias de libre disposición conforme a derecho, así como aquellas que la ley o los tratados o acuerdos internacionales autoricen.

2. Cuando el arbitraje sea internacional y una de las partes sea un Estado o una sociedad, organización o empresa controlada por un Estado, esa parte no podrá invocar las prerrogativas de su propio derecho para sustraerse a las obligaciones derivadas del convenio arbitral. 
Como se puede ver el arbitraje en Perú tiene una base muy fuerte que busca su cumplimiento una vez que se la haya elegido, por lo que utiliza todos los mecanismos necesarios para que este se realice. Busca ensanchar las materias que pueden arbitrarse al no tener una lista taxativa, sino abierta, aunque sigue manteniendo las materias que son estructurales para el orden social dentro de la competencia de la justicia ordinaria.

En conclusión, los criterios de arbitrabilidad pueden variar de país a país, pero de manera general se puede ver que todos mantienen una misma línea general, y es que buscan abrirse al arbitraje, pero sin dejar de lado el orden y la seguridad necesarios en la estructura social de un país y de una comunidad; es por todo esto que el legislador de cada país decide resguardar las materias más delicadas para que se resuelva en sus propias manos.

\section{LA TRANSIGIBILIDAD Y SU PROBLEMÁTICA}

En el Ecuador, el criterio acogido por la normativa respecto de la arbitrabilidad de la materia en el proceso arbitral corresponde al de la transigibilidad [supra §5.]. Para MARCHÁN ${ }^{73}$ esta disposición no generaría mayor novedad con la emisión de la CRE en el año 2008, pues es el mismo criterio que acoge la $\mathrm{LAM}^{74}$. No obstante, es momento de mencionar toda la problemática que este criterio ha demostrado durante los veinte y dos años de vigencia de la LAM y su ratificación en la CRE.

En primer lugar, la regla no aclara cuáles serían las materias susceptibles de transacción, a criterio propio, esto enreda más el sistema. Es por ello, que para doctrinarios como PARRAQUEZ \& DARQUEA, se debe interpretar y entender la transigibilidad por prohibición o descarte, es decir que: "será transigible todo

73. J. M. MARChÁN, "El tratamiento de arbitraje en la nueva Constitución ecuatoriana", Iuris Dictio, Vol. 14, p. 204.

74. Ley de Arbitraje y Mediación, N. 15, Art. 1. 
aquello que no haya sido declarado expresamente como no transigible por ley"75. Ahora bien, que sucede cuando existe duda o el legislador no ha regulado determinados temas. Esto, a todas luces, complica el sistema, ya que limita el uso del arbitraje como un MASC efectivo.

Esto se puede evidenciar en las diferentes ramas del derecho en que han existido controversia respecto de su arbitrabilidad o no. De manera ejemplificativa, se puede enunciar la materia laboral ${ }^{76}$, ciertas situaciones derivadas de materia administrativa ${ }^{77}$, otras relacionadas con el derecho de competencia ${ }^{78}$ o aquellos que sostienen que la materia tributaria podría ser susceptible de arbitraje ${ }^{79}$.

En segundo lugar, y como consecuencia del anterior, el criterio queda en desuso cuando la ley no regula todos los supuestos en los que se puede considerar como transigible determinada materia. Esto genera como consecuencia que, además del criterio de transigibilidad, se utilice y sea necesario otro razonamiento. Según CAIVANO, respecto a temas de arbitrabilidad objetiva, se suele utilizar el argumento de 'normas de orden público' para determinar que existen temas que no son susceptibles de arbitraje $\mathrm{e}^{80}$. Es decir, el sistema de análisis se complica y se fragmenta, toda vez que los errores son más proclives de aparecer y contagiar el correcto desarrollo del proceso arbitral. Ya que esto generaría que el tribunal arbitral se encuentre obligado a inhibirse de conocer la controversia por falta de competencia.

75. L. Parraguez \& J. C. Darquea, "La arbitrabilidad del daño moral", Revista Ecuatoriana de Arbitraje, No. 8, 2016, p. 97.

76. M. E. JARA, "Reflexiones sobre el arbitraje como mecanismo alternativo para la solución de conflictos individuales de trabajo", Revista Ecuatoriana de Arbitraje, No. 7, 2015, p. 40.

77. J. P. Agullar, "Derecho Administrativo y Transigibilidad". Revista Ecuatoriana de Arbitraje, No. 6, 2014, p. 250.

78. F. Pozo, "La Relación entre el Derecho de la Competencia y el Arbitraje en Ecuador", Revista Ecuatoriana de Arbitraje, No. 7, 2015, p. 303.

79. M. Flor, "El Arbitraje Tributario Interno", Revista Ecuatoriana de Arbitraje, No. 2, 2010, p. 122.

80. R. J. Caivano, N. 49, p. 8. 
En tercer lugar, la transigibilidad como regla necesaria para determinar la materia que puede ser arbitrada es totalmente incorrecta. El proceso arbitral busca verificar la existencia o no de un determinado derecho. El arbitraje es un MASC heterocompositivo que se resuelve sobre la base del criterio de un tercero imparcial. En el caso concreto, el tribunal arbitral deberá decidir la solución de la controversia con base en los hechos del caso y la subsunción de estos en la norma, si se trata de arbitraje en derecho; o deberá resolver sobre la base de su leal saber y entender cuando se trate de arbitrajes en equidad.

Es claro que, en ningún caso el proceso arbitral involucra una disposición, negociación o renuncia de derechos, al ser un proceso de conocimiento lo que se busca es esclarecer el conflicto y darle una solución. Por lo tanto, se demuestra que el criterio de transigibilidad es utilizado de manera incorrecta, toda vez que la materia discutida no debería ser susceptible de negociación.

A lo largo del estudio propuesto de arbitrabilidad objetiva, se puede llegar a la conclusión que los criterios que se utilizan, tanto en la legislación ecuatoriana como en el derecho comparado son comunes y en ciertos aspectos similares. Además, se verifica que, respecto a la arbitrabilidad de la materia, la política legislativa y el orden público juegan un papel sumamente importante. En palabras de CENTENO, MorAles y SÁNCHEZ, "sin duda alguna se pueden cometer errores al momento de determinar la competencia en razón de la materia con el ejercicio de esta valoración" ${ }^{81}$. Por lo tanto, se reafirma la intención de cambiar el razonamiento de arbitrabilidad en Ecuador. Por lo tanto, el criterio arbitrabilidad debe ser delimitado por el legislador, sin embargo, debe realizarlo de manera consciente y adecuada al desarrollo del arbitraje actualmente.

81. V. Centeno, A. Morales \& R. I. SÁnchez, "Falta de competencia del Tribunal Arbitral como causal de nulidad del laudo", Revista Ecuatoriana de Arbitraje, No. 9, 2017. 
Bajo ningún concepto, se pretende instaurar un mecanismo absoluto y totalmente símil a la justicia ordinaria, ya que se parte de la idea que el arbitraje es una excepción, por lo tanto, debe existir materias de carácter no arbitrables. Pero es preciso aclarar que no puede existir dudas respecto a la arbitrabilidad de estas. Por esta razón, se propone eliminar de manera absoluta el criterio de transigibilidad, así como olvidar definitivamente el de renunciabilidad. Estos serán reemplazados por la voluntad y criterio tota de la política legislativa, es decir solo debe existir prohibición de arbitrabilidad en la ley. De esta manera se solucionarían todos los conflictos, y largos análisis, de la materia.

\section{A manera de CONClusión}

En el arbitraje se debe tener en cuenta una división de momentos jurídicos más que momentos temporales en sí mismos. Este iter está compuesto por la: (i) Existencia; (ii) Validez; y, (iii) Suficiencia del convenio arbitral. Siguiendo esta línea, es importante destacar las leyes que rigen al arbitraje son: ley procesal o lex arbitrii, ley sustancial y ley proccidental. Este criterio es preciso aclarar, toda vez que se debe acoger los mandatos de la ley sustantiva para el análisis del iter de la cláusula arbitral.

En primer lugar, para considerar que el convenio arbitral existe se deben completar los requisitos; (i) voluntad; (ii) el objeto; y, (iii) las solemnidades que la ley exija. Dentro de este análisis se puede considerar a la escrituración de la convenio arbitral como un requisito de prueba, más que un elemento ad solemnitaten. Continuando con este análisis, para que sea válido el convenio arbitral debe tener los siguientes requisitos: (i) capacidad de las partes; (ii) voluntad libre de vicios; (iii) objeto lícito; y (iv) causa lícita. Por último, la suficiencia o eficacia del convenio se analiza para que surta los efectos deseados por las 
partes, debido a que este se traduce como la competencia del tribunal arbitral para poder pronunciarse o inhibirse acerca de un determinado conflicto. Esto con base en el principio de kompetenz-kompetenz es la potestad del tribunal para conocer su propia competencia y autoridad.

Teniendo en cuenta la competencia del tribunal arbitral, se puede determinar que existen 3 fases de análisis, la competencia (i) ratione voluntatis; (ii) ratione personae; $y$, (iii) ratione materiae. Profundizando en el objetivo trabajo, llegamos al desarrollo y definición de la arbitrabilidad; esta tiene que ver con la aptitud del tribunal arbitral para conocer y pronunciarse respecto de un determinado conflicto. La arbitrabilidad se divide en subjetiva y objetiva; la primera trata sobre los sujetos, los cuales pueden o no llevar sus controversias a arbitraje; mientras que, la segunda se refiere a la materia puede o no ser objeto de arbitraje.

El objetivo de este trabajo es demostrar que existen tendencias en la actualidad sobre algunas materias que pueden llegar a ser objeto de arbitraje, como a su vez se pueden encontrar bifurcaciones en las propias tendencias que toma cada derecho doméstico respecto a un tema específico. Los criterios de arbitrabilidad pueden variar de país a país, así como en la tendencia internacional, pero de manera general se puede ver que todos mantienen una misma línea general, y es que buscan abrirse al arbitraje, pero sin dejar de lado el orden y la seguridad necesarios en la estructura social de un país y de una comunidad. Por esta razón, el legislador de cada país decide resguardar las materias más delicadas para que se resuelva en sus propias manos.

Tras la expedición de la LAM han existido varios temas de análisis y desarrollo que han surgido con la evolución práctica del sistema arbitral. Sin embargo, queda claro que varios aspectos aún deben ser analizados y reformados para 
evitar futuros inconvenientes como los que han aparecido en los últimos años.

El primer criterio por destacar es que transigibilidad no es lo mismo que renunciabilidad. Estas pueden presentar conflictos y confusiones debido a la evolución histórica de la arbitrabilidad objetiva en el Ecuador. Actualmente, el criterio a seguir respecto de la competencia ratione materiae es la transigibilidad de la materia. Sin embargo, de ello, esta postura instaurada en la CRE y la LAM genera problemas.

La problemática que se encuentra es la siguiente: (i) la regla no aclara cuáles serían las materias susceptibles de transacción; (ii) el criterio queda en desuso cuando la ley no regula todos los supuestos en los que se puede considerar como transigible determinada materia, generando la necesidad de otro razonamiento; y, (iii) la transigibilidad como regla necesaria para determinar la materia que puede ser arbitrada es totalmente incorrecta. Es absurdo sostener que para arbitrar sea necesario poder disponer de derechos, ya que el proceso arbitral busca verificar la existencia o no de un determinado derecho, bajo ningún concepto involucra la negociación o la mediación del conflicto. Se puede afirmar que un proceso arbitral es lo más parecido a juicio en justicia ordinaria, por lo que esto involucraría que para litigar en la función judicial también sería necesario ser capaz de transigir los derechos que se cuestionan.

Una vez demostrada la problemática que engloba la transigibilidad como arbitrabilidad objetiva, es necesario mostrar una solución al respecto. Los criterios que se utilizan, tanto en la legislación ecuatoriana como en el derecho comparado son comunes y en ciertos aspectos similares. El criterio de arbitrabilidad debe ser delimitado por el legislador, sin embargo, debe realizarlo de manera consciente y adecuada al desarrollo del arbitraje actual. Por lo que, lo más correcto es que exista una limitación expresa de todas las situaciones 
inarbitrables, para asegurar la aplicación del principio de la seguridad jurídica, y así, evitar interpretaciones extendidas o bloqueos a la evolución del arbitraje.

Finalmente, se propone eliminar de manera absoluta el criterio de transigibilidad, así como olvidar definitivamente el de renunciabilidad. Estos serán reemplazados por la voluntad y criterio total de la política legislativa, es decir, sólo debe existir prohibición de arbitrabilidad en la ley. De esta manera, en un futuro, el arbitraje nacerá verdaderamente completo y listo para el beneficio de todos quienes acuden a él, como fue el caso de Atenea; y, en un momento dado llegar a ser el favorito de Zeus, la justicia ordinaria. 